\title{
Antiferromagnetic four-dimensional O(4) model
}

\author{
Isabel Campos* \\ Departamento de Física Teórica, Facultad de Ciencias, Universidad de Zaragoza, 50009 Zaragoza, Spain \\ Luis A. Fernández $z^{\dagger}$ \\ Departamento de Física Teórica I, Facultad de Ciencias Físicas, Universidad Complutense de Madrid, 28040 Madrid, Spain \\ Alfonso Tarancón \\ Departamento de Física Teórica, Facultad de Ciencias, Universidad de Zaragoza, 50009 Zaragoza, Spain
}

(Received 2 July 1996)

\begin{abstract}
We study the phase diagram of the four-dimensional $\mathrm{O}(4)$ model with first- $\left(\beta_{1}\right)$ and second- $\left(\beta_{2}\right)$ neighbor couplings, especially in the $\beta_{2}<0$ region, where we find a line of transitions which seems to be second order. We also compute the critical exponents on this line at the point $\beta_{1}=0$ ( $F_{4}$ lattice) by finite-size scaling techniques up to a lattice size of 24 , these exponents being different from the mean-field ones. [S0556-2821(97)04205-7]
\end{abstract}

PACS number(s): 11.10.Lm, 05.70.Fh, 12.40.Ee, 75.10.Hk

\section{INTRODUCTION}

The action for the electroweak sector of the standard model has $\mathrm{SU}(2) \times \mathrm{U}(1)$ symmetry. If we consider the $\mathrm{SU}(2)$ part and take the limit in which gauge degrees of freedom are frozen, the resulting action is the $\mathrm{O}(4)$ nonlinear $\sigma$ model, which has been extensively studied because its spontaneous symmetry breaking pattern is equivalent to the one exhibited by $\mathrm{SU}(2)$ in four dimensions [1]. The regularized version of the $\mathrm{O}(4) \sigma$ model on the lattice leads to an interacting continuum limit for $d<4$ [2], while for $d>4$ the theory is described by free bosonic fields $[3,4]$.

At the upper critical dimension $d=4$, deviations from mean-field theory (MFT) are expected. The MFT predictions for the scaling of thermodynamic quantities are corrected by multiplicative logarithmic terms [5,6].

Perturbatively the infrared fixed point of the CallanSymanzik function $\beta(g)$ moves to the origin as the dimension becomes four [5], also the fixed point is now a double zero (in contrast with the $d<4$ case) which is responsible for the occurrence of such logarithmic corrections.

The existence of these corrections implies the triviality of the theory [7]. Triviality seems to persist when gauge fields are included $[8,9]$.

The common feature of all these approaches to the socalled triviality problem [8], is that the self-interactions of the scalar field in the broken phase are weak, and they can be reasonably studied within the context of perturbation theory. It is generally believed that the perturbative and the strongcoupling regime belong to the same universality class. However, for the nonperturbative strong-coupling regime a rigorous proof is still lacking and we have to rely on numerical simulations $[10,11]$.

The existence of a strongly interacting Higgs sector, with

\footnotetext{
*Electronic address: isabel@ sol.unizar.es

†Electronic address: laf@lattice.fis.ucm.es

${ }^{\ddagger}$ Electronic address: tarancon@sol.unizar.es
}

a complicated dynamics, rendering useless perturbation theory, is a possibility not to be discarded a priori. A large amount of work has actually been done in order to know whether or not nonperturbative effects could change the physics of the electroweak symmetry breaking sector (for a review see [12]). In this sense, concerning the universality class of the $R P^{N-1}$ models, the role of nonperturbative effects needs to be clarified [14].

Antiferromagnetism (AF) has been considered in a great variety of models in order to find properties not present in the purely ferromagnetic (FM) systems $[13,15]$. In the context of high- $T_{c}$ superconductivity, AF seems to play an essential role. The transition from paramagnetic to nonpurely FM ordered phases has been studied in two-dimensional models [16-19].

In four dimensions, in diluted systems recently new critical exponents have been obtained [20]. Also in $d=4$ a previous study of the AF Ising model [21] shows the existence of an AF phase nontrivially equivalent to the standard FM one. However, no new critical behavior was evidenced in this work.

Also in four dimensions, competing interactions have been considered in order to study the multicritical point of the Yukawa models. At this multicritical point four phases meet [FM, AF, ferrimagnetic, and paramagnetic (PM)]. The question of whether or not it would be possible to define a nontrivial continuum limit at this point still remains an open problem [22-25].

It is not clear the role that AF can play in the formulation of quantum field theory (QFT), nevertheless a careful study of these kinds of models is worthwhile since they are known to have very rich phase diagrams, and presumably new universality classes could appear in which alternative formulations of continuum QFT should be possible. However, when defining a theory with AF couplings one has to be aware of the fact that higher order derivatives tends to violate reflection positivity [26,27]. A possibility is to perform an appropriate tuning of the couplings in order to cancel the contributions coming from unphysical (negative norm) states. 
The inclusion of gauge fields can change the situation [27], but it is worthwhile as a probe to see what happens in this limit when negative couplings are included, postponing for a future study the effect of gauge fields. In this work we study how the existence of opposite couplings influence the vacuum of the theory, specifically, whether or not the ground state $(\Omega)$ is frustrated (the energy cannot be minimized simultaneously for all couplings) or even disordered (nonzero vacuum entropy).

\section{THE MODEL}

Our starting point is the nonlinear $\sigma$ model, with the action

$$
S_{\sigma}=-\beta \sum_{\mathbf{r}, \mu} \boldsymbol{\Phi}_{\mathbf{r}} \boldsymbol{\Phi}_{\mathbf{r}+\hat{\mu}},
$$

where $\boldsymbol{\Phi}$ is a four-component vector with fixed modulus $\boldsymbol{\Phi}_{\mathbf{r}} \cdot \boldsymbol{\Phi}_{\mathbf{r}}=1$.

The naive way to introduce $\mathrm{AF}$ in the nonlinear $\sigma$ model is to consider a negative coupling. In this case the state with minimal energy for large $\beta$ is a staggered vacuum. On a hypercubic lattice, if we denote the coordinates of site $\mathbf{r}$ as $\left(r_{x}, r_{y}, r_{z}, r_{t}\right)$, making the transformation

$$
\boldsymbol{\Phi}_{\mathbf{r}} \rightarrow(-1)^{r_{x}+r_{y}+r_{z}+r_{t}} \boldsymbol{\Phi}_{\mathbf{r}},
$$

the system with negative $\beta$ is mapped onto the positive $\beta$ one, both regions being exactly equivalent.

Therefore to consider true AF we must take into account either different geometries or more couplings, in order to break the symmetry under the transformation (2). In four dimensions the simplest option is to add more couplings, we have chosen to add a coupling between points at a distance of $\sqrt{2}$ lattice units.

Following this we will consider a system of spins $\left\{\boldsymbol{\Phi}_{\mathbf{r}}\right\}$ taking values in the hypersphere $S^{3} \subset \mathbf{R}^{4}$ and placed in the nodes of a cubic lattice. The interaction is defined by the action

$$
S=-\beta_{1} \sum_{\mathbf{r}, \mu} \boldsymbol{\Phi}_{\mathbf{r}} \Phi_{\mathbf{r}+\hat{\mu}}-\beta_{2} \sum_{\mathbf{r}, \mu<\nu} \boldsymbol{\Phi}_{\mathbf{r}} \Phi_{\mathbf{r}+\hat{\mu}+\hat{\nu}} .
$$

The transformation (2) maps the semiplane $\beta_{1}>0$ onto the $\beta_{1}<0$, and therefore only the region with $\beta_{1} \geqslant 0$ will be considered. On the line $\beta_{1}=0$ the system decouples in two $F_{4}$ independent sublattices.

When $\beta_{2}=0$ the model is known to present a continuous transition between a disordered phase, where $\mathrm{O}(4)$ symmetry is exact, to an ordered phase where the $\mathrm{O}(4)$ symmetry is spontaneously broken to $\mathrm{O}(3)$. This transition is second order, being the critical exponents those of MFT: $\alpha=0$, $\nu=0.5, \beta=0.5, \eta=0$, and $\gamma=1$ up to logarithmic corrections. The critical coupling for this case can be studied analytically by an expansion in powers of the coordination number $(q=2 d)$, being $\beta^{\mathrm{c}}=0.6055+O\left(q^{-2 d}\right)[28]$.

From a mean-field analysis, we observe that for $\beta_{2}>0$ the behavior of the system will not change qualitatively from the $\beta_{2}=0$ case but with higher coordination number. In fact, taking into account that the energy (for nonfrustrated sys- tems) is approximately proportional to the coordination number, there will be a transition phase line whose approximate equation is

$$
\beta_{1}^{\mathrm{c}}+q \beta_{2}^{\mathrm{c}}=\beta^{\mathrm{c}},
$$

where $q$ is the quotient between the number of second and first neighbors. This line can be thought of as a prolongation of the critical point at $\beta_{2}=0$ so the transitions on this line are expected to be second order with MFT exponents. This is also the behavior of the two couplings Ising model in this region [21]. When $\beta_{2}<0$, the presence of two couplings with opposite sign makes frustration appear, and very different vacua are possible.

\section{OBSERVABLES AND ORDER PARAMETERS}

We define the energy associated to each coupling:

$$
\begin{gathered}
E_{1} \equiv \frac{\partial \ln Z}{\partial \beta_{1}}=\sum_{\mathbf{r}, \mu} \boldsymbol{\Phi}_{\mathbf{r}} \cdot \boldsymbol{\Phi}_{\mathbf{r}+\hat{\mu}}, \\
E_{2} \equiv \frac{\partial \ln Z}{\partial \beta_{2}}=\sum_{\mathbf{r}, \mu<\nu} \boldsymbol{\Phi}_{\mathbf{r}} \cdot \boldsymbol{\Phi}_{\mathbf{r}+\hat{\mu}+\hat{\nu}} .
\end{gathered}
$$

In terms of these energies, the action reads

$$
S=-\beta_{1} E_{1}-\beta_{2} E_{2} .
$$

It is useful to define the energies per bound as

$$
e_{1}=\frac{1}{4 V} E_{1}, \quad e_{2}=\frac{1}{12 V} E_{2},
$$

where $V=L^{4}$ is the lattice volume. With this normalization $e_{1}, e_{2}$ belong to the interval $[-1,1]$.

We have computed the configurations which minimize the energy for several asymptotic values of the parameters. We have only considered configurations with periodicity two. More complex structures have not been observed in our simulations.

Considering only the $\beta_{1} \geqslant 0$ case, we have found the following regions.

(1) Paramagnetic (PM) phase or disordered phase, for small absolute values of $\beta_{1}, \beta_{2}$.

(2) Ferromagnetic (FM) phase. It appears when $\beta_{1}+6 \beta_{2}$ is large and positive.

When the fluctuations go to zero, the vacuum takes the form $\boldsymbol{\Phi}_{\mathbf{r}}=\mathbf{v}$, where $\mathbf{v}$ is an arbitrary element of the hypersphere.

Concerning the definition of the order parameter let us remark that because of tunneling phenomena in finite lattice we are forced to use pseudo-order parameters for practical purposes. Such quantities behave as true order parameters only in the thermodynamical limit. In the FM phase, we define the standard (normalized) magnetization as

$$
\mathbf{M}_{\mathrm{F}}=\frac{1}{V} \sum_{\mathbf{r}} \boldsymbol{\Phi}_{\mathbf{r}},
$$

and we use as pseudo-order parameter the square root of the norm of the magnetization vector 


$$
M_{\mathrm{F}}=\left\langle\sqrt{\mathbf{M}_{\mathrm{F}}^{2}}\right\rangle .
$$

This quantity has the drawback of being nonzero in the symmetric phase but it presents corrections to the bulk behavior order $1 / \sqrt{V}$.

(3) Hyperplane antiferromagnetic (HPAF) phase. It corresponds to large $\beta_{1}$, with $\beta_{2}$ in a narrow interval ( $\left[-\beta_{1} / 2,-\beta_{1} / 6\right]$ in the mean-field approximation). In this region the vacuum correspond to spins aligned in three directions but antialigned in the fourth $(\mu)$.

In absence of fluctuations the associated vacuum would be $\boldsymbol{\Phi}_{\mathbf{r}}=(-1)^{r}{ }^{\mu} \mathbf{v}$, where $\mu$ can be any direction, and $\mathbf{v}$ any vector on $\mathrm{S}^{4}$. We define an ad hoc order parameter for this phase as

$$
\mathbf{M}_{\mathrm{HPAF}, \mu}=\frac{1}{V} \sum_{\mathbf{r}}(-1)^{r} \mu \mathbf{\Phi}_{\mathbf{r}} .
$$

$\mathbf{M}_{\mathrm{HPAF}, \mu}$ will be different from zero only in the HPAF phase, where the system becomes antiferromagnetic on the $\mu$ direction. From the four order parameters (one for every possible value of $\mu$ ) only one of them will be different from zero in the HPAF phase. So, we define the pseudo-order parameter as

$$
M_{\mathrm{HPAF}}=\sqrt{\sum_{\mu} \mathbf{M}_{\mathrm{HPAF}, \mu}^{2}} .
$$

(4) Plane antiferromagnetic (PAF) phase for $\beta_{2}$ large and negative. In this region the ground state is a configuration with spins aligned in two directions and antialigned in the remaining two. It is characterized by one of the six combinations of two different directions $(\mu, \nu)$, and an arbitrary spin v: $\boldsymbol{\Phi}_{\mathbf{r}}=(-1)^{r_{\mu}+r_{\nu}}$. For the PAF region we first define

$$
\mathbf{M}_{\mathrm{PAF}, \mu, \nu}=\frac{1}{V} \sum_{r}(-1)^{r_{\mu}+r_{\nu}} \boldsymbol{\Phi}_{\mathbf{r}},
$$

and the quantity we measure is

$$
M_{\mathrm{PAF}}=\sqrt{\sum_{\mu<\nu} \mathbf{M}_{\mathrm{PAF},(\mu, \nu)}^{2}} .
$$

In order to avoid undesirable (frustrating) boundary effects for ordered phases, we work with even lattice side $L$ as periodic boundary conditions are imposed.

From this data we can compute the derivatives of any observable with respect to the couplings as the connected correlation function with the energies

$$
\frac{\partial O}{\partial \beta_{j}}=\left\langle O E_{j}\right\rangle-\langle O\rangle\left\langle E_{j}\right\rangle .
$$

An efficient method to determine $\beta_{\mathrm{c}}$ for a second order transition is to measure the Binder cumulant [29] for various lattice size and to locate the cross point in the space of $\beta$.

For $\mathrm{O}(N)$ models $U_{L}(\beta)$ takes the form [30]

$$
U_{L}(\beta)=1+2 / N-\frac{\left\langle\left(\mathbf{m}^{2}\right)^{2}\right\rangle}{\left\langle\mathbf{m}^{2}\right\rangle^{2}},
$$

where $\mathbf{m}$ is an order parameter for the transition. It can be shown $[29,30]$ that $U_{L}(0) \rightarrow O(1 / V)$ and $U_{L}(\infty) \rightarrow 2 / N$. The slope of $U_{L}(\beta)$ at $\beta_{\mathrm{c}}$ increases with $L$. The value of the Binder cumulant is closely related with the triviality of the theory since the renormalized coupling (in the massless thermodynamical limit) at zero momentum can be written as

$$
g_{\mathrm{R}}=\lim _{L \rightarrow \infty} g_{\mathrm{R}}(L)=\lim _{L \rightarrow \infty}\left(L / \xi_{L}\right)^{d} U_{L}\left(\beta_{\mathrm{c}}\right)
$$

where $\xi_{L}$ is the correlation length in the size $L$ lattice.

From this point of view triviality is equivalent to have a vanishing $g_{\mathrm{R}}$ in the thermodynamical limit. In this context it is clear that we can use the value of $g_{\mathrm{R}}$ to classify the universality class. Out of the upper critical dimension, $L / \xi_{L}$ is a constant at $\beta_{\mathrm{c}}$ since $\xi \sim L$, and we could use the Binder cumulant for the same purpose [31]. At the upper critical dimension, $\xi_{L}$ presents logarithmic corrections and $L / \xi_{L}$ is no longer a constant at $\beta_{\mathrm{c}}$. For the $\mathrm{FM} \mathrm{O}(4)$ model in $d=4$ (upper critical dimension) we have perturbatively $L / \xi_{L} \sim(\ln L)^{-1 / 4}$ [32]. In order to have a nontrivial theory, the Binder cumulant should behave as a positive power of $\ln L$, but from its definition [29] we see that $U_{L}(\beta) \leqslant 1$. This is just another way of stating the perturbative triviality of the FM O(4) model.

\section{SYMMETRIES ON THE $F_{4}$ LATTICE}

In the $\beta_{1}=0$ case the system decouples in two independent lattices, each one constituted by the first neighbors of the other. So we consider two lattices with $F_{4}$ geometry. There are several reasons to choose the point $\beta_{1}=0$ for a careful study of the PM-PAF transition. The region with $\beta_{1}>1.5$ evolves painfully with our local algorithms; for small $\beta_{1}$ we expect a very large correlation in Monte Carlo (MC) time because the interaction between both sublattices is very small, and the response of one lattice to changes in the other is very slow. We also remark that the presence of two almost decoupled lattices is rather unphysical. We have also the experience from a previous work for the Ising model [21] that the correlation length at its first order transition is smaller in the $F_{4}$ lattice, that means, we can find asymptotic critical behavior in smaller lattices.

However we should point out that the results in the $F_{4}$ lattice cannot be easily extrapolated to a neighborhood of the $\beta_{1}$ axis. Certainly, the geometry of the model is very modified when $\beta_{1} \neq 0$, and perhaps continuity arguments present problems. Nevertheless, we have run also the case $\beta_{1} \sim 0$, and as occurs in the Ising model we have not found qualitative differences. In the following when we refer to the size of the lattice $L$ on the $F_{4}$ lattice we mean a lattice with $L^{4} / 2$ sites.

We have to find the configurations that maximize $E_{2}$ in order to define appropriate order parameters for the phase transition. The system has a very complex structure. As a starting point we have studied numerically the vacuum with $\beta_{2} \ll 0$. For this values we have found in the simulation.

(1) The vacuum has periodicity two. To check this, we have defined 


$$
\mathbf{V}_{i}=\frac{1}{L^{d} / 2^{d}} \sum_{I} \boldsymbol{\Phi}_{I_{i}}
$$

where $i=0, \ldots, 7$ stands for the $i$ th vertex of each $2^{4}$ hypercube belonging to the $F_{4}$ lattice, and with $I$ we denote the $2^{4}$ hypercubes themselves.

From these vectors we can define the eight magnetizations associated to the elementary cell,

$$
V_{i}=\left\langle\sqrt{\mathbf{V}_{i}^{2}}\right\rangle
$$

We have checked that all $V_{i}$ tends to 1 for the ordered phase in the thermodynamical limit, so we conclude that the ordered vacua have periodicity two. Let us remark for the sake of completeness that all order parameters we have defined can be written as an appropriate linear combination of the $\mathbf{V}_{i}$.

(2) In the elementary cell, $\boldsymbol{\Phi}_{\mathbf{r}+\hat{\mu}+\hat{\nu}}=\boldsymbol{\Phi}_{\mathbf{r}} \forall \mu, \nu$ with $\mu<\nu$. So, in this section we will restrict the study of the vacuum structure to the four sites $(i=0,1,2,3)$ belonging to the cube in the hyperplane $r_{t}=0$.

(3) We have measured the energy per bound associated to the second-neighbor coupling. We check that in the thermodynamical limit $e_{2}=-1 / 3$.

(4) If we choose the symmetry breaking direction by keeping fixed one vector (e.g., $\boldsymbol{\Phi}_{0}$ ), we find

$$
\sum_{i=1}^{3}\left[\left(\boldsymbol{\Phi}_{0} \cdot \boldsymbol{\Phi}_{\mathbf{i}}\right) \boldsymbol{\Phi}_{0}-\boldsymbol{\Phi}_{\mathbf{i}}\right]=0
$$

The vacuum structure is not completely fixed by these three conditions since different symmetry breaking patterns are possible. For instance, a configuration $\boldsymbol{\Phi}_{0}=(1,0,0,0)$, $\boldsymbol{\Phi}_{1}=\left[-1 / 3,(2 \sqrt{2} / 3) \mathbf{v}_{1}\right], \quad \boldsymbol{\Phi}_{0}=\left[-1 / 3,(2 \sqrt{2} / 3) \mathbf{v}_{\mathbf{2}}\right], \quad \boldsymbol{\Phi}_{0}$ $=\left[-1 / 3,(2 \sqrt{2} / 3) \mathbf{v}_{3}\right]$, with $v_{i}$ a three-component unitary vector with the constraint $\sum_{i \neq j} \mathbf{v}_{\mathbf{i}} \mathbf{v}_{\mathbf{j}}=0$, breaks $\mathrm{O}(4)$, but an $\mathrm{O}(2)$ symmetry remains (for the different $v_{i}$ ).

To determine which is the vacuum in presence of fluctuations, we consider four independent fields in a $2^{4}$ cell with periodic boundary conditions. Let us first consider an $\mathrm{O}(2)$ group. We can study the four vectors as a mechanical system of massless links of length unity, rotating in a plane around the same point, whose extremes are attached with a spring of natural length zero. The energy for the system is

$$
E=-\sum_{i, j=0, i>j}^{3} \cos \left(\theta_{i}-\theta_{j}\right)
$$

We consider the fluctuation matrix, $H=\partial E^{2} / \partial \theta_{i} \partial \theta_{j}$ in order to find the normal modes. The matrix elements of $H$ take the form

$$
H_{i, j}=\delta_{i j} \sum_{k \neq i} \cos \left(\theta_{i}-\theta_{k}\right)-\cos \left(\theta_{i}-\theta_{j}\right)\left(1-\delta_{i j}\right)
$$

In the FM case the minimum corresponds to $\theta_{i}=\phi$, for all $i$. There is a single zero mode, and a three times degenerated nonzero mode with eigenvalue $\lambda=-4$.

For the AF (maximum energy) case, the maximum energy is found, up to permutations, at $\theta_{0}=\phi, \theta_{1}=\phi+\pi$, $\theta_{2}=\phi+\alpha$, and $\theta_{3}=\phi+\pi+\alpha, \forall \alpha$. In addition to the $\phi$ freedom that corresponds to the global $\mathrm{O}(2)$ symmetry, there is a degeneration of the vacuum in the $\alpha$ angle and this zero mode is double $\forall \alpha$. The other two eigenvalues are $\lambda_{1,2}=2(1 \pm \cos \alpha)$, so, an additional zero mode appears when $\alpha=0$, obtaining in this case a threefold degenerated zero mode corresponding to $\theta_{0}=\theta_{1}=\theta_{2}+\pi=\theta_{3}+\pi$.

The $\mathrm{O}(4)$ case is qualitatively similar. We have 12 degrees of freedom. Of all configurations that minimize the energy, that with a largest degeneration (nine times) consist of two spins aligned and two antialigned that correspond to a PAF vacuum. We consider this degeneration as the main difference with the FM sector, and could be relevant to obtain different critical exponents.

In the presence of fluctuations the configurations with largest degeneration are favored by phase space considerations, so we expect that the real vacuum is a PAF one. This statement will be checked below with Monte Carlo data in the critical region.

\section{FINITE-SIZE SCALING (FSS) ANALYSIS}

Our measures of critical exponents are based on the FSS ansatz $[33,34]$. Let the mean value of an observable measured on a size $L$ lattice at a coupling $\beta$ be $\langle O(L, \beta)\rangle$. If $O(\infty, \beta) \sim\left|\beta-\beta_{\mathrm{c}}\right|^{x_{O}}$, from the FSS ansatz one readily obtains [34]

$$
\langle O(L, \beta)\rangle=L^{x_{O} / \nu} F_{O}(L / \xi(\infty, \beta))+\cdots,
$$

where $F_{O}$ is a smooth function and the dots stand for corrections to scaling terms.

To obtain $\nu$ we apply Eq. (23) to the operator $d \ln M_{\mathrm{PAF}} / d \beta$ whose related $x$ exponent is 1 . As this operator is almost constant in the critical region, we just measure at the extrapolated critical point or any definition of the apparent critical point in a finite lattice, the difference being small corrections-to-scaling terms.

For the magnetic critical exponents the situation is more involved as the slope of the magnetization or the unconnected susceptibility is very large at the critical point. We proceed as follows (see Refs. [13] for other applications of this method). Let $\Theta$ be any operator with scaling law $x_{\Theta}=1$ (for instance the Binder parameter or a correlation length defined in a finite lattice divided by $L$ ). Applying Eq. (23) to an arbitrary operator $O$ and $\Theta$ we can write

$$
\langle O(L, \beta)\rangle=L^{x_{O} / \nu} f_{O, \Theta}(\langle\Theta(L, \beta)\rangle)+\cdots
$$

Measuring the operator $O$ in a pair of lattices of sizes $L$ and $s L$ at a coupling where the mean value of $\Theta$ is the same, one readily obtains

$$
\left.\frac{\langle O(s L, \beta)\rangle}{\langle O(L, \beta)\rangle}\right|_{\Theta(L, \beta)=\Theta(s L, \beta)}=s^{x_{O} / \nu}+\cdots .
$$

The use of the spectral density method (SDM) [35] avoids an exact a priori knowledge of the coupling where the mean values of $\Theta$ cross. We remark that usually the main source of statistical error in the measures of magnetic exponents is the error in the determination of where to measure the coupling. However, using Eq. (25) we can take into account the corre- 
lation between the measures of the observable and the measure of the coupling where the cross occurs. This allows to reduce the statistical error in an order of magnitude.

\section{FSS AT THE UPPER CRITICAL DIMENSION: LOGARITHMIC CORRECTIONS}

It is well known that $d=4$ is the upper critical dimension of the FM O(4) model. As we have already pointed out logarithmic corrections to the mean-field predictions are expected. In particular, FSS in its standard formulation breaks at $d=4$ because the essential assumption, namely $\xi_{L}\left(\beta_{\mathrm{c}}\right) \sim L$ is no longer true. In fact, in four dimensions [7]

$$
\xi(\infty, t) \sim|t|^{-1 / 2}|\ln | t||^{1 / 4} .
$$

The FSS formula for the correlation length was calculated by Brezin [32]. At the critical point one gets

$$
\xi\left(L, \beta_{\mathrm{c}}\right) \sim L(\ln L)^{1 / 4} .
$$

It has been suggested [10] that the usual FSS statement should be replaced by the more general

$$
\frac{O\left(L, \beta_{\mathrm{c}}\right)}{O(\infty, \beta)}=F_{O}\left(\frac{\xi\left(L, \beta_{\mathrm{c}}\right)}{\xi(\infty, \beta)}\right) .
$$

When applying the quotient method described above to systems in four dimensions one has to take into account the logarithmic corrections, so that the modified formula reads

$$
\left.\frac{\langle O(s L, \beta)\rangle}{\langle O(L, \beta)\rangle}\right|_{\Theta(L, \beta)=\Theta(s L, \beta)}=s^{x_{O} / \nu}\left(1+\frac{\ln s}{\ln L}\right)^{1 / 4} .
$$

This point is particularly important when measuring the magnetic critical exponents because as we have already mentioned, the slope of the magnetization and susceptibility are very large, and one has to be very careful where to measure when locating the coupling.

\section{NUMERICAL METHOD}

We have simulated the model in a $L^{4}$ lattice with periodic boundary conditions. The biggest lattice size has been $L=24$. For the update we have employed a combination of heat-bath and over-relaxation algorithms (ten over-relax sweeps followed by a heat-bath sweep).

The dynamic exponent $z$ we obtain is near 1 . Cluster-type algorithms are not expected to improve this $z$ value. In systems with competing interactions the cluster size average is a great fraction of the whole system, losing the efficacy they show for ferromagnetic spin systems.

We have used for the simulations ALPHA-processorbased machines. The total computer time employed has been the equivalent of two years of ALPHA AXP3000. We measure every 10 sweeps and store the individual measures to extrapolate in a neighborhood of the simulation coupling by using the SDM.

In the $\mathrm{F}_{4}$ case, we have run about $2 \times 10^{5} \tau$ for each lattice size, $\tau$ being the largest integrated autocorrelation time measured, that corresponds to $M_{\mathrm{PAF}}$, and ranges from 2.3 measures for $L=6$ to 8.9 for $L=24$. We have discarded more

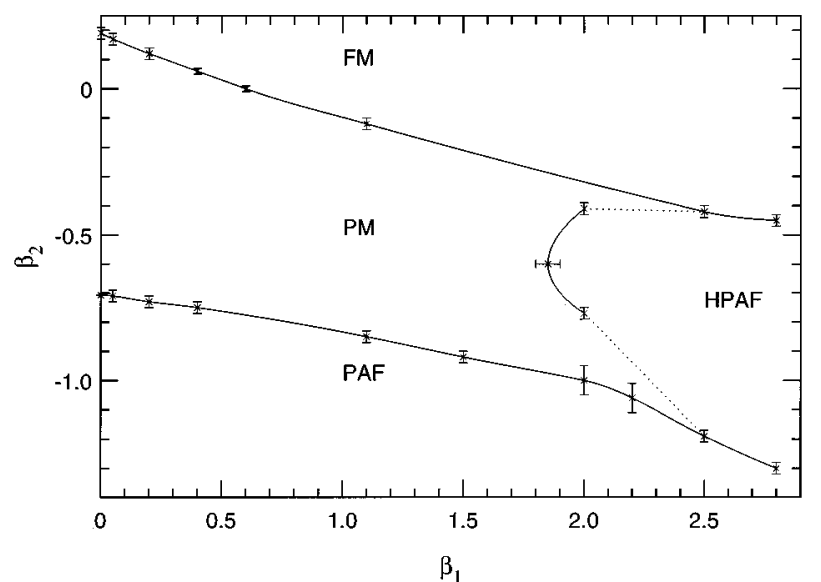

FIG. 1. Phase diagram obtained from the MC simulation on a $L=8$ lattice.

than $10^{2} \tau$ for thermalization. The errors have been estimated with the jackknife method.

\section{RESULTS AND MEASURES}

\section{A. Phase diagram}

We have studied the phase diagram of the model using a $L=8$ lattice. We have done a sweep along the parameter space of several thousands of iterations, finding the transition lines shown in Fig. 1. The symbols represent the coupling values where a peak in the order parameter derivative appears.

The line FM-PM has a clear second-order behavior. It contains the critical point for the $\mathrm{O}(4)$ model with firstneighbor couplings $\left(\beta_{1} \approx 0.6, \beta_{2}=0\right)$ with classical exponents $(\nu=0.5, \eta=0)$. In the $\beta_{1}=0$ axis, we have computed the critical coupling $\left(\beta_{2}^{\mathrm{c}} \approx 0.18\right)$ and the critical exponents as a test for the method in the $F_{4}$ lattice. We have also considered the influence of the logarithmic corrections when computing the exponents. The lines FM-HPAF, HPAF-PAF, and PM-HPAF show clear metastability, indicating a first order transition.

The regions between the lower dotted line and the PAF transition line, and between the upper dotted line and the FM transition line, are disordered up to our numerical precision. We could expect always a PM region separating the different ordered phases, however, from a MC simulation it is not possible to give a conclusive answer since the width of the hypothetical PM region decreases when increasing $\beta_{1}$, and for a fixed lattice size there is a practical limit in the precision of the measures of critical values.

On the line PM-PAF we have found no signs of first order. We have done hysteresis cycles in several points and no metastability has been observed. In Fig. 2 we plot the energy distribution at the coupling where a peak in the specific heat appears. There is no evidence of two-state signal up to $L=24$.

The likely second-order behavior of the PM-PAF transition line contrasts with the first order one found in the Ising model with two couplings in the analogous region [21]. This is not surprising because we are dealing now with a global continuous symmetry. The spontaneous symmetry breaking 


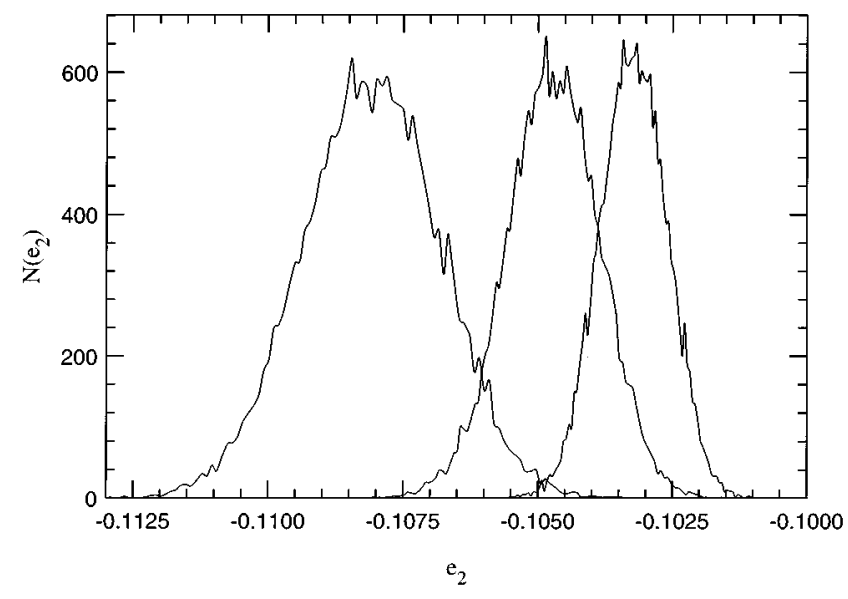

FIG. 2. Energy distribution for $L=16,20$, and 24 in the $F_{4}$ lattice at the peak of the specific heat.

of such symmetries manifests in the appearance of soft modes or low-energy excitations (long wavelength), the Goldstone bosons in QFT terminology [36]. The role of these soft modes is quite important and is actually under a a vigorous discussion in the two-dimensional case $[37,38]$. In general, these low-energy modes will perturb the mechanism of long-distance ordering, softening in this way the phase transitions.

Regarding the differences with the FM case, the most remarkable feature is the different vacuum structures appearing, especially the very large degeneration in the PAF transition, in contrast with the single degeneration of the FM $\mathrm{O}(4)$ mode. As a simpler point for study the properties of the transition, namely the critical exponents, is the $F_{4}$ limit, most of the MC work has been done for this case.

\section{B. Results on the $F_{4}$ lattice \\ 1. Results on the FM region}

First, we have checked our method on the FM region of the $F_{4}$ lattice. In Fig. 3 the crossing points of the Binder cumulant for various lattice sizes are displayed. The prediction for the critical coupling $\beta_{\mathrm{c}} \sim 0.1831(1)$ agrees with an earlier study by Bhanot [39].

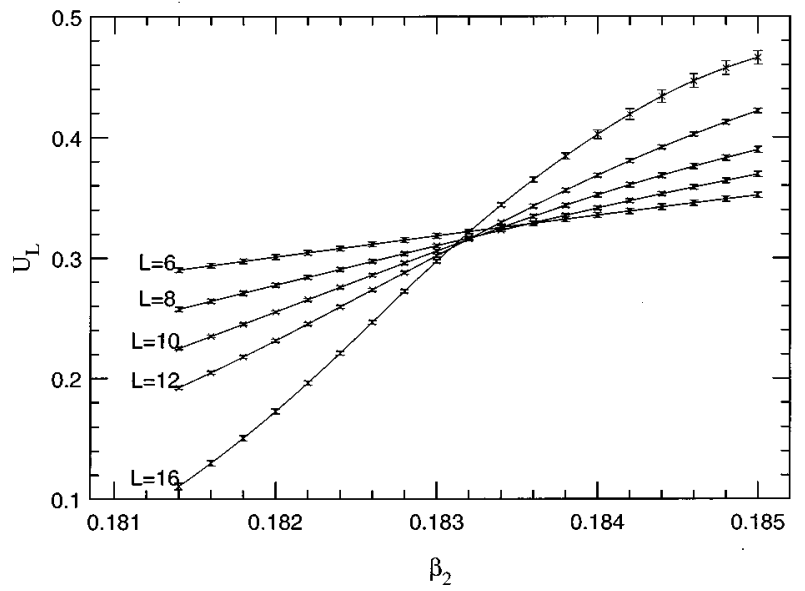

FIG. 3. Crossing points of the Binder cumulant for various lattice sizes on the transition FM-PM.
TABLE I. Critical exponents for the FM-PM phase transition in the $F_{4}$ lattice.

\begin{tabular}{lccc}
\hline \hline$L$ values & $8 / 16$ & $12 / 16$ & $10 / 12$ \\
\hline \multicolumn{4}{c}{ (without logarithmic corrections) } \\
$\nu$ & $0.483(8)$ & $0.495(4)$ & $0.467(26)$ \\
$\beta / \nu$ & $0.92(3)$ & $0.94(3)$ & $0.87(4)$ \\
$\gamma / \nu$ & $2.16(2)$ & $2.12(2)$ & $2.24(4)$ \\
& (with logarithmic corrections) \\
$\nu / \nu$ & $0.512(6)$ & $0.509(7)$ & $0.492(10)$ \\
$\gamma / \nu$ & $1.04(3)$ & $1.06(3)$ & $1.04(2)$ \\
\hline \hline
\end{tabular}

Concerning the measures of critical exponents, we have applied the quotient method, described in Sec. IV. In Table I we quote the results when logarithmic corrections are included [formula (29)], and also for sake of comparison, when they are neglected [formula (25)]. The results obtained with logarithmic correction do not rule out the possibility of nonlogarithmic corrections, but point out in correct direction, making the exponents to be closer to the mean-field ones. From now on we will focus on the transition between the PM phase and the PAF phase on the $F_{4}$ lattice.

\section{Vacuum symmetries on the PAF region}

We will check using MC data that the ordered vacuum in the critical region is of type PAF.

Let us define

$$
A_{i j}=\mathbf{V}_{i} \cdot \mathbf{V}_{j}
$$

The leading ordering corresponds to the eigenvector associated to the maximum eigenvalue of the matrix $A$, that should scale as $L^{-2 \beta / \nu}$ at the critical point. The scaling law of the biggest eigenvalue agrees with the $\beta / \nu$ value reported in Table III, and the associated eigenvector is, within errors, $(1,1,-1,-1)$.

We also have found that the other eigenvalues scale as $L^{-4}$. This is the expected behavior if just the $\mathrm{O}(4)$ symmetry is broken, and it remains an $\mathrm{O}(3)$ symmetry in the subspace orthogonal to the $\mathrm{O}(4)$ breaking direction.

\section{Critical coupling}

To obtain a precise determination of the critical point $\beta_{\mathrm{c}}$ we have used the data for the Binder parameter (16). In Fig. 4 we plot the crossing points of the Binder cumulants for the simulated lattices sizes. Extrapolations have been done using SDM from simulations at $\beta_{2}=-0.7090$ for $L=6,8,10,12$, and $16 ; \beta_{2}=-0.7078$ for $L=20$, and $\beta_{2}=-0.7070$ for $L=24$.

The shift of the crossing point of the curves can be explained through the finite-size confluent corrections. The dependence in the deviation of the crossing point for $L$ and $s L$ size lattices was estimated by Binder [29]

$$
\beta_{\mathrm{c}}(L, s L)-\beta_{\mathrm{c}} \sim \frac{1-s^{-\omega}}{s^{1 / \nu}-1} L^{-\omega-1 / \nu},
$$




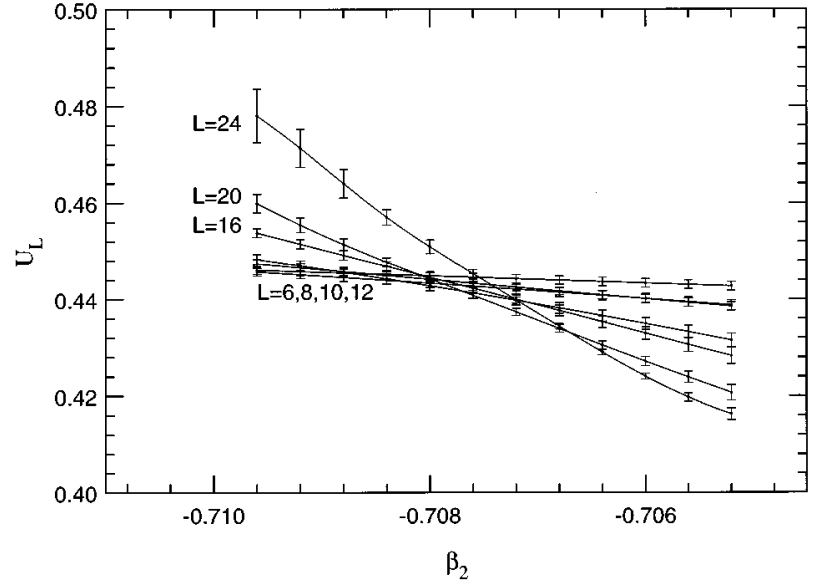

FIG. 4. Crossing points of the Binder cumulant for various lattice sizes on the transition PM-PAF.

where $\omega$ is the universal exponent for the corrections to scaling.

The infinite volume critical point the value

$$
\beta_{\mathrm{c}}=-0.7065(5)[+2][-2],
$$

where the errors in brackets correspond to the variations in the extrapolation when we use the values $\omega=0.5$ and $\omega=2$, respectively. In Fig. 5 we plot Eq. (31) for $\omega=1$.

Using the previous value of $\beta_{\mathrm{c}}$ we can compute the Binder cumulant at this point. In Table II we quote the obtained values. The result points to that the Binder cumulant stays constant in the critical region. This result would be compatible with a nonzero value of the renormalized coupling when $L$ increases. Concerning the possibility of having logarithmic corrections in the determination of the critical coupling, from the numerical point of view, it is not possible to discern between the $\omega$ effect, and a logarithmic correction.

\section{Thermal critical exponents $\alpha, \nu$}

The critical exponent associated to correlation length can be obtained from the scaling of

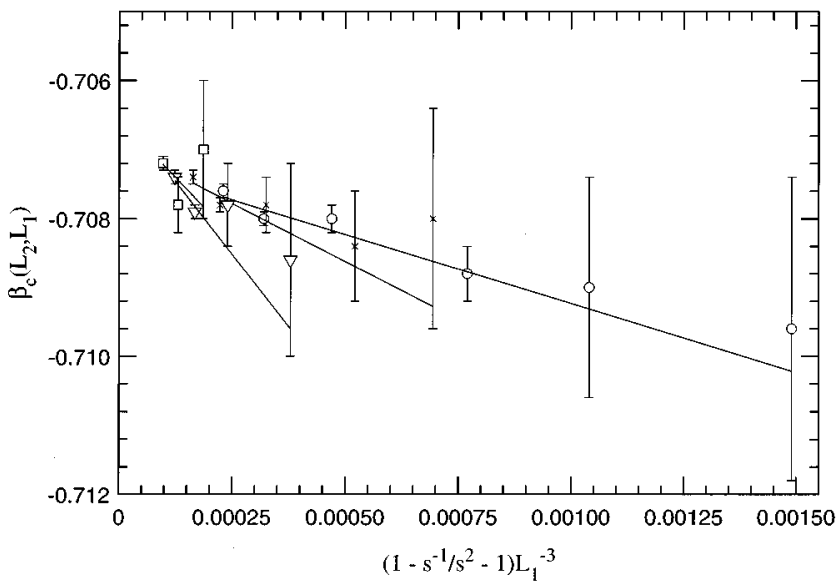

FIG. 5. Extrapolation to $\beta_{\mathrm{c}}(\infty)$ for $L_{1}=6,8,10,12$ (circle, cross, triangle, and square symbols, respectively).
TABLE II. Binder cumulant for various lattices sizes at the extrapolated critical point for $\omega=0.5,1,2$.

\begin{tabular}{lccc}
\hline \hline Lattice sizes & $U_{L}\left[\beta_{\mathrm{c}}(\omega=0.5)\right]$ & $U_{L}\left[\beta_{\mathrm{c}}(\omega=1)\right]$ & $U_{L}\left[\beta_{\mathrm{c}}(\omega=2)\right]$ \\
\hline 6 & $0.4435(15)$ & $0.4437(12)$ & $0.4438(12)$ \\
8 & $0.4406(15)$ & $0.4409(12)$ & $0.4413(12)$ \\
10 & $0.4407(14)$ & $0.4411(16)$ & $0.4414(14)$ \\
12 & $0.436(4)$ & $0.437(3)$ & $0.438(3)$ \\
16 & $0.435(3)$ & $0.436(3)$ & $0.437(3)$ \\
20 & $0.429(5)$ & $0.431(5)$ & $0.433(5)$ \\
24 & $0.428(6)$ & $0.430(7)$ & $0.433(7)$ \\
\hline \hline
\end{tabular}

$$
\kappa=\frac{\partial \ln M}{\partial \beta},
$$

where $M$ is an order parameter for the transition, $M_{\mathrm{PAF}}$ for our purposes. In the critical region $\kappa \sim L^{1 / \nu}$. As $\kappa$ is a flat function of $\beta$, the point where we actually measure is not crucial. The results displayed in Table III have been obtained measuring at the crossing point of the Binder parameters for lattice sizes $L$ and $2 L$ using Eq. (25).

For measuring $\alpha / \nu$ we study the scaling of the specific heat

$$
C=\frac{\partial\left\langle E_{2}\right\rangle}{\partial \beta_{2}}
$$

We expect that $C$ scales as $A+B L^{\alpha / \nu}$, where $A$ is usually non-negligible. In Fig. 6 we plot the specific heat measuring at Eq. (32), as well as at the peak of the specific heat, as a function of $L$. We observe a linear behavior for intermediate lattices. For the largest lattice the slope decreases. The weak first order behavior [40] $(\alpha / \nu=1$ for small lattices that becomes $d$ for large enough sizes) seem hardly compatible with our data. If we neglect the $A$ term (which is asymptotically correct), and compute the exponent using Eq. (25) we obtain $\alpha / \nu \approx 0.3$ for intermediate lattices that reduces to $\alpha / \nu=0.15(2)$ for the $(20,24)$ pair. However, it is mandatory to give a conclusive answer for the value of $\alpha$ statistics on larger lattices.

TABLE III. Critical exponents for the PM-PAF phase transition in the $F_{4}$ lattice.

\begin{tabular}{lccc}
\hline \hline Lattice sizes & $\gamma / \nu$ & $\beta / \nu$ & $\nu$ \\
\hline \multicolumn{4}{c}{ (without logarithmic corrections) } \\
$6 / 12$ & $2.417(3)$ & $0.791(4)$ & $0.474(10)$ \\
$8 / 16$ & $2.403(3)$ & $0.792(6)$ & $0.483(8)$ \\
$10 / 20$ & $2.410(2)$ & $0.790(4)$ & $0.471(6)$ \\
$12 / 24$ & $2.403(5)$ & $0.797(5)$ & $0.483(7)$ \\
$20 / 24$ & $2.398(5)$ & $0.802(4)$ & $0.487(6)$ \\
& (with logarithmic corrections) \\
$6 / 12$ & $2.301(3)$ & $0.849(4)$ & $0.484(9)$ \\
$8 / 16$ & $2.300(3)$ & $0.850(5)$ & $0.489(7)$ \\
$10 / 20$ & $2.315(2)$ & $0.843(3)$ & $0.488(5)$ \\
$12 / 24$ & $2.314(5)$ & $0.842(5)$ & $0.487(5)$ \\
$20 / 24$ & $2.317(5)$ & $0.839(4)$ & $0.498(5)$ \\
\hline \hline
\end{tabular}




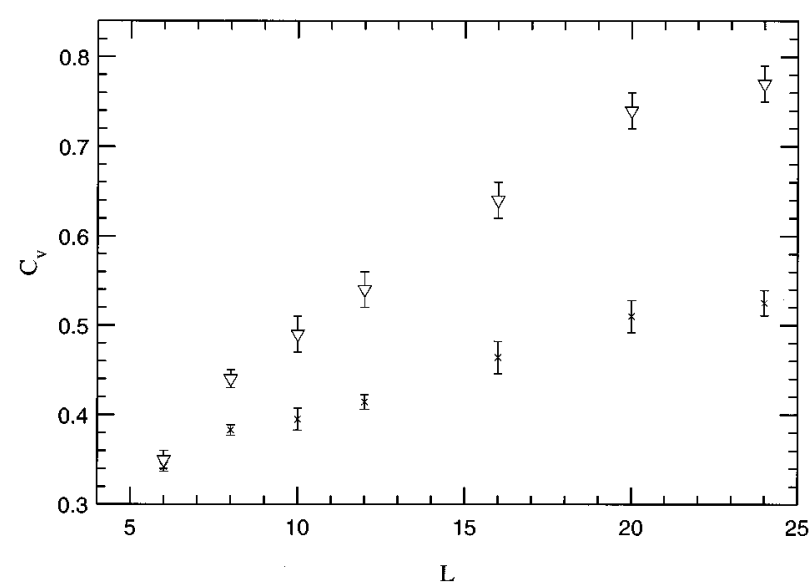

FIG. 6. Specific heat at the peak (triangle symbols) and at $\beta=-0.7068$ (cross symbols) as a function of the lattice size.

\section{Magnetic critical exponents $\gamma, \beta$}

The exponents $\gamma$ and $\beta$ can be obtained, respectively, from the scaling of susceptibility and magnetization:

$$
\begin{gathered}
\chi \equiv V\left\langle M^{2}\right\rangle \sim L^{\gamma / \nu}, \\
M \sim L^{-\beta / \nu},
\end{gathered}
$$

where $M$ is an order parameter for the phase transition. In Fig. 7 upper part, we plot the quotient between $M_{\mathrm{PAF}}$ for lattices $L$ and $2 L$ as a function of the quotient between the Binder cumulants for both lattice sizes.

For large $L$ in the critical region we should obtain a single curve, the deviations corresponding to corrections to scaling. In the lower part of Fig. 7 we plot the same function for susceptibility. The values for $\gamma$ and $\beta$ are summarized in Table III.

\section{Logarithmic corrections}

We now address the question of the possibility of logarithmic corrections in the $\mathrm{AF} \mathrm{O}(4)$ model. For the thermal critical exponents, the situation seems clear, they are compatible with the classical exponent $\nu=0.5$. For the magnetic exponents, the situation is more involved. In principle, one can think that they disagree from MFT due to logarithmic

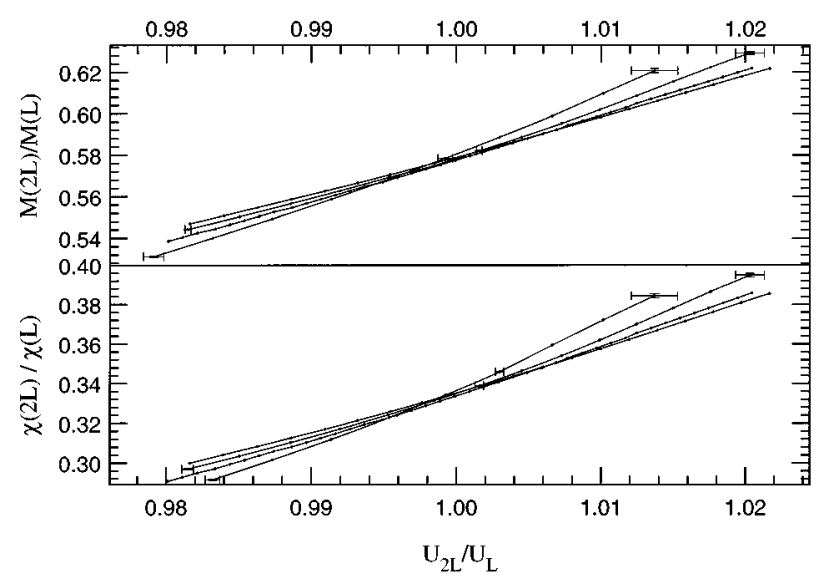

FIG. 7. Quotients to obtain $\beta / \nu$ and $\gamma / \nu$. corrections. We have no perturbative predictions about the form in which these corrections would affect $\xi_{L}$ for the AF case. However, one expects that such corrections slightly modify the critical exponents, as occurs in the FM case. It could be possible that logarithmic corrections modify largely the previous critical exponents and drift them to the FM ones. To sort this out, we have considered the possibility of a FM-like behavior, so that $\xi_{L} \sim L(\ln L)^{1 / 4}$. In the lower part of Table III we quote the values of the critical exponents for the PAF phase transition when logarithmic corrections are included [formula (29)]. We see how in effect the magnetic critical exponents are too far from the classical ones for being the result of a logarithmic correction to the MFT predictions. It is interesting to compare this situation with that in the $\mathrm{RP}^{2}$ model in $d=4$ [15] where small deviations from MFT exponents can be explained as logarithmic corrections.

\section{CONCLUSIONS AND OUTLOOK}

We have studied the phase diagram of the fourdimensional $\mathrm{O}(4)$ model with first- and second-neighbor couplings. For $\beta_{2}<0$ we find a region nontrivially related with the FM one, in which the system is AF ordered in some plane. The phase transition between the disordered region and this PAF region seems to be second order.

We also compute the critical exponents on this line at $\beta_{1}=0$ ( $F_{4}$ lattice $)$ by means of FSS techniques. We found that up to $L=24$ the exponents are in disagreement with the mean-field predictions. Specifically, from our $\gamma / \nu$ estimation (or $\beta / \nu$ using the hyperscaling relation) the exponent $\eta$ associated with the anomalous dimension of the field is $\eta \approx-0.4$. This fact itself would imply the nontriviality of the theory because Green functions would not factorize anymore. One cannot discard that the observed behavior were transitory. However, the stability of our measure of $\gamma / \nu$ for lattice sizes ranging from $L=6$ to $L=24$, which are more than a hundred standard deviations apart in the MF value, makes this hypothesis very unlikely. Actually, it would be possible to obtain triviality also with a logarithmic exponent in Eq. (29) different from 1/4. We can fix the critical exponents to its MF value and compute this parameter from the numerical data. The results obtained show a nonasymptotic behavior, with values ranging from 0.8 to 1.2 for the lattices used. A logarithmic fit is not satisfactory because of the nonasymptoticity and the large value of the logarithmic exponent, but larger lattices sizes are needed in order to get a more conclusive answer.

The behavior of the specific heat does not show any first order signature, but we have not been able to obtain a reliable estimation of the $\alpha$ exponent. We have also measured the Binder cumulant at the critical point, finding that it stays almost constant when increasing the lattice size. If this is not a transient effect, and logarithmic corrections are finally ruled out, it would correspond to a nonzero value of the renormalized constant in the thermodynamical limit.

\section{ACKNOWLEDGMENTS}

The authors are grateful to J. L. Alonso, H. G. Ballesteros, V. Martín-Mayor, J. J. Ruiz-Lorenzo, C. L. Ullod, and D. Iniguez for helpful discussions and comments. We thank es- 
pecially J. M. Carmona for useful discussions concerning the logarithmic corrections. One of us (I.C.) wishes to thank R. D. Kenway for this kind hospitality at the Department of Physics and Astronomy, University of Edinburgh, as well as to Edinburgh Parallel Computing Center (EPCC) for com- puting facilities where part of these computations have been done under the financial support of TRACS-EC program. This work was partially supported by CICyT AEN95-1284, and AEN96-1670. I.C. thanks the Ministerio de Educación y Ciencia for financial support.
[1] S. Weinberg, Phys. Rev. Lett. 19, 1264 (1967).

[2] D. Brydges, J. Frohlich, and T. Spencer, Commun. Math. Phys. 83, 123 (1982).

[3] M. Aizenman, Phys. Rev. Lett. 47, 1 (1981).

[4] J. Frolich, Nucl. Phys. B200 [FS4], 281 (1982).

[5] E. Brezin, J. C. Le Guillou, and J. Zinn-Justin, in Phase Transitions and Critical Phenomena, edited by C. Domb and M. S. Green (Academic, London 1976).

[6] F. J. Wegner and E. K. Riedel, Phys. Rev. B 7, 248 (1973).

[7] M. Luscher and P. Weisz, Nucl. Phys. B299 [FS20], 25 (1987).

[8] D. E. Callaway, Nucl. Phys. B233, 189 (1984).

[9] RTN Collaboration, J. L. Alonso et al., Nucl. Phys. B405, 575 (1993).

[10] R. Kenna and C. B. Lang, Nucl. Phys. B393, 461 (1993).

[11] R. Kenna and C. B. Lang, in Lattice '92, Proceedings of the International Symposium, Amsterdam, The Netherlands, edited by J. Smit and P. van Baal [Nucl. Phys. B (Proc. Suppl.) 30, 697 (1993)].

[12] I. Montvay, in Lattice '91, Proceedings of the International Symposium, Tsukuba, Japan, edited by M. Fukugita et al. [Nucl. Phys. B, (Proc. Suppl.) 26, 57 (1991)].

[13] H. G. Ballesteros, L. A. Fernández, V. Martín-Mayor, and A. Muñoz-Sudupe, Phys. Lett. B 378, 207 (1996); Nucl. Phys. (to be published).

[14] E. Seiler and K. Yildirim, Report No. hep-lat/ 9609030 (unpublished).

[15] H. G. Ballesteros, J. M. Carmona, L. A. Fernández, V. MartínMayor, A. Muñoz-Sudupe and A. Tarancón, Report No. DFTUZ 96/20 (unpublished).

[16] M. L. Plumer and A. Caillé, J. Appl. Phys. 70, 5961 (1991).

[17] H. Kawamura, J. Phys. Soc. Jpn. 61, 1299 (1992).

[18] H. Mori and M. Hamada, Physica B 194-196, 1445 (1994).

[19] J. L. Morán-López, F. Aguilera-Granja, and J. M. Sánchez, J. Phys. Condens. Matter 6, 9759 (1994).

[20] G. Parisi and J. J. Ruiz-Lorenzo, J. Phys. A 28, L395 (1995).
[21] J. L. Alonso, J. M. Carmona, J. Clemente, L. A. Fernández, D. Iniguez, A. Tarancón, and C. L. Ullod, Phys. Lett. B 376, 148 (1996).

[22] W. Bock, A. K. De, K. Jansen, J. Jersak, T. Neuhaus, and J. Smit, Nucl. Phys. B344, 207 (1990).

[23] W. Bock, A. K. De, C. Frick, J. Jersak, and T. Trapenberg, Nucl. Phys. B378, 652 (1992).

[24] T. Ebihara and K. Kondo, Prog. Theor. Phys. 87, 4 (1992).

[25] J. L. Alonso, Ph. Boucaud, F. Lesmes, and E. Rivas, Phys. Lett. B 329, 75 (1994).

[26] G. Gallavotti and V. Rivasseau, Phys. Lett. 122B, 268 (1983).

[27] Jochen Fingberg and Janos Polonyi, Report No. hep-lat/ 9602003 (unpublished).

[28] P. R. Gerber and M. E. Fisher, Phys. Rev. B 10, 4697 (1974).

[29] K. Binder, Z. Phys. B 43, 119 (1981).

[30] E. Brezin and J. Zinn-Justin, Nucl. Phys. B257 [FS14], 867 (1995).

[31] Giorgio Parisi, Statistical Field Theory (Addison-Wesley, New York, 1992).

[32] E. Brezin, J. Phys. (France) 43, 15 (1982).

[33] C. Itzykson and J. M. Drouffe, Statistical Field Theory (Cambridge University Press, Cambridge, England, 1989), Vol. 1.

[34] Finite size scaling, Current Physics-Sources and comments, edited by J. L. Cardy (Elsevier, New York, 1988).

[35] A. M. Ferrenberg and R. H. Swendsen, Phys. Rev. Lett. 61, 2635 (1988).

[36] Michel Le Bellac, Des Phenomenes Critiques Aux Champs de Jauge (Inter-Editions/Editions du CNRS, Paris, 1988).

[37] B. Alles, A. Buonanno, and G. Cella, in Lattice '96, Proceedings of the International Symposium, St. Louis, Missouri, edited by T. Golterman [Nucl. Phys. B, Proc. Suppl. (in press)], Report No. hep-lat/9609030 (unpublished).

[38] E. Seiler and A. Patrasciou, Report No. hep-lat/ 9608138 (unpublished).

[39] G. Bhanot, Nucl. Phys. B, Proc. Suppl. 17, 653 (1990).

[40] L. A. Fernández, M. P. Lombardo, J. J. Ruiz-Lorenzo, and A. Tarancón, Phys. Lett. B 277, 485 (1992). 